tungszeiten nach UV-Bestrahlung und für die 3-Stdn.Bebrütungszeit nach Röntgenbestrahlung (s. Abbn. $4 \mathrm{a}-\mathrm{c}$ und $5 \mathrm{a}$ ) bezeugt, daß ein gleicher Prozentsatz der Inaktivierung der Koloniebildungsfähigkeit beider Stämme stets zu der gleichen Inaktivierung der Syntheseraten beider Stämme führt. Entscheidend für die Hemmung der DNS-Syntheserate ist demnach nicht die applizierte absolute Strahlendosis, sondern die bezogen auf die Koloniebildungsfähigkeit wirksam werdende Dosis.

Abweichend hiervon verhalten sich die Werte für DNS-Syntheseraten und Koloniebildungsvermögen beider Stämme nach Röntgenbestrahlung und 6 bzw. 9 Stdn. Bebrütungszeit ( $5 \mathrm{~b}$ und c). Das bereits in 3. festgestellte gegenläufige Verhalten der Hemmung der DNS-Syntheseraten beider Stämme verursacht dieses Ergebnis. Ohne im einzelnen schon heute interpretierbar zu sein, können diese Ergebnisse als Hinweis auf einen unterschiedlichen Ablauf von Reparaturprozessen nach UV- und Röntgenbestrahlung in Zellen des Stammes 291 gedeutet werden.

Auf Unterschiede im Reparaturvermögen strahleninduzierter Schädigungen sind die Unterschiede in der Koloniebildungsfähigkeit beider Stämme zurückzuführen (s. l. c. ${ }^{2}$ ). Bei gleicher applizierter Strahlendosis kann Stamm 291 mehr strahleninduzierte Schädigungen reparieren als Stamm 298. Die bei Stamm 291 erfolgten Reparaturen ermöglichen eine höhere Koloniebildungsrate und eine höhere DNSSyntheserate als bei Stamm 298. Wie bereits in l. c. ${ }^{1}$ diskutiert, wird in Übereinstimmung mit PAINTER und RASMUSSEN ${ }^{5}$ der zweiphasige Verlauf der DEK durch die Annahme verständlich, daß eine maximale Syntheserate nur bei Vorhandensein ungestörter großer DNS-Abschnitte, die in HeLa-Zellen wenigstens ein Mol.-Gew. von $10^{9}-10^{10}$ haben, möglich ist. Falls diese Annahme zutrifft, folgt, daß die in Stamm 291 ablaufenden Reparaturprozesse zur Wiederherstellung großer ungestörter DNS-Abschnitte führen. Welcher Art die durch die Reparatur beseitigten Störungen sind, bleibt allerdings noch ungeklärt.

Für technische Asistenz sind wir Frau I. MensenDIEK zu Dank verpflichtet.

\title{
Die Wirkung einiger Orotaldehyd-Derivate als Orotsäureantagonisten bei Neurospora Crassa
}

\author{
Evgeny Golovinsky, Alexander Spassov und Elisaveta Roussinova \\ Zentrales Biochemisches Laboratorium der Bulgarischen Akademie der Wissenschaften \\ Sofia, Bulgarien
}

(Z. Naturforschg. 24 b, 1315-1317 [1969] ; eingegangen am 29. April 1969)

\begin{abstract}
Es wird die Wirkung des Orotaldehydoxims, des Orotaldehydthiosemicarbazons, des Orotaldehydsemicarbazons und des Orotaldehydphenylhydrazons als potentielle Antimetabolite der Orotsäure auf das Wachstum von Neurospora crassa untersucht. Die höchste wachstumshemmende Wirkung zeigt das Orotaldehydthiosemicarbazon. Der wuchshemmende Effekt der untersuchten Orotaldehyd-Derivate wird durch Orotsäure enthemmt.
\end{abstract}

In der letzten Zeit ist das Interesse an der Synthese und dem Wirkungsmechanismus von Orotsäureantimetaboliten merkbar gestiegen. Manche dieser Verbindungen zeigen eine geschwulsthemmende Aktivität (5-Azaorotsäure, 5-Fluororotsäure u. a.) .

In der vorliegenden Arbeit wird die Wirkung einiger Orotaldehyd-Derivate, und zwar des Orotaldehydoxims (1), des Orotaldehydthiosemicarbazons (2), des Orotaldehydsemicarbazons (3) und des Orotaldehydphenylhydrazons (4), als potentielle
Orotsäureantimetabolite auf Neurospora crassa untersucht.

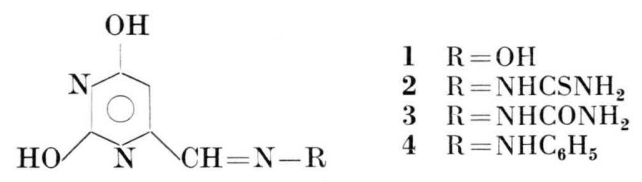

Wenn man das Atommodell der Orotsäure mit dem des Orotaldehydoxims vergleicht, so stellt man cine große Ähnlichkeit im Ausmaß und im räum- 
lichen Aufbau beider Molekel fest. Außerdem ist die Acidität der Aldoximgruppe geringer als die der Carboxylgruppe. Auf Grund dieser Voraussetzungen kann angenommen werden, daß das Orotaldehydoxim und möglicherweise auch andere OrotaldehydDerivate als Orotsäureantagonisten wirken können.

Die Chemie des Orotaldehyds ist noch wenig erforscht. Erst in der letzten Zeit wurde eine einfache Methode zur Herstellung dieses Aldehyds ausgearbeitet, die in der Oxydation des 6-Methyluracils mittels Selendioxid besteht ${ }^{1,2}$. Für unsere Versuche haben wir diese Methode verwendet. Es sind auch wenige Orotaldehyd-Derivate bekannt ${ }^{2,3}$.

\section{Experimenteller Teil}

Orotaldehydoxim (1). Nach Erwärmen von 1, $58 \mathrm{~g}$ $(0,01 \mathrm{Mol})$ Orotaldehyd und $0,70 \mathrm{~g}$ Hydroxylaminhydrochlorid in Gegenwart von $3,0 \mathrm{ml}$ Pyridin (wäßrige Lösung) beträgt die Ausbeute an reinem Orotaldehydoxim $0,64 \mathrm{~g}$ (42 Prozent). Bei schnellem Erhitzen $\left(3-4^{\circ} /\right.$ Min.) zersetzt sich die Substanz bei $262-265^{\circ}$. Wenn sie langsamer erhitzt wird, zeigt sie keinen definierten Zersetzungspunkt, sondern verkohlt allmählich ab $210^{\circ}$. Die Reinigung des Produktes wurde auch spektrophotometrisch verfolgt. Es ergaben sich farblose Prismen (aus Wasser).

$\mathrm{C}_{5} \mathrm{H}_{5} \mathrm{O}_{3} \mathrm{~N}_{3}(155,11)$

Ber. N 27,09; Gef. N 26,65, 26,62.

Orotaldehydthiosemicarbazon (2), Orotaldehydsemicarbazon (3) und Orotaldehydphenylhydrazon (4). Diese Verbindungen wurden nach l. c. ${ }^{2,3}$ synthetisiert und gereinigt. Sie sind sehr wenig löslich in Wasser und anderen Lösungsmitteln.

Horizontalwuchstest mit Neurospora grassa. Wir haben den Horizontalwuchstest nach RAUEN ${ }^{4}$ verwen. det. Die Versuche wurden mit dem Neurospora-Stamm ATCC $9863 *$ ausgeführt.

Bei den Versuchen wurden nur gut gereinigte Präparate sowie eine Orotsäure von der Fa. E. Merck, Darmstadt, verwendet. Die wenig wasserlöslichen Orotaldehyd-Derivate 2, 3 und $\mathbf{4}$ wurden in Form einer Suspension getestet.

Bei den Untersuchungen über die enthemmenden Wirkungen der Orotsäure auf den wuchshemmenden Effekt des Orotaldehydoxims und des Orotaldehydthiosemicarbazons verwandten wir diese Orotaldehyd-Derivate in einer Konzentration von $1 \mu \mathrm{mMol} / \mathrm{ml}$. Um die Wirkung höhrer Konzentrationen zu enthemmen, sind

1 I. A. Mickailopulo, V. I. Gunar u. S. I. Zavjalov, Ber. Akad. Wiss. UdSSR. 1967, 1388.

2 Kwang-Yuen Zee-Cheng u. C. C. Cheng, J. Heterocyclic Chem. 4, 163 [1967].

3 M. Claessen u. H. Vanderhaeghe, Bull. Soc. chem. Belgique 66, 292 [1957]. größere Mengen von Orotsäure zu verwenden, die unter den Versuchsbedingungen schon zum Teil ungelöst bleiben.

Die angegebenen Werte der Hemmung bzw. Enthemmung sind Mittelwerte von 3 bis 5 Versuchen. Der Variationskoeffizient jedes Mittelwertes betrug 5-14 Prozent.

\section{Ergebnisse und Diskussion}

Die untersuchten Orotaldehyd-Derivate hemmen das Wachstum von Neurospora crassa. Hemmtyp a2cI nach l.c. ${ }^{4}$. Die hemmende Wirkung des Orotaldehydoxims und des Thiosemicarbazons ist auf Abb. 1 wiedergegeben. Wie zu sehen ist, übt das Orotaldehydthiosemicarbazon eine höhere Hemmung auf das Neurospora-Wachstum aus als das Orotaldehydoxim. Die anderen Orotaldehyd-Derivate sind weniger aktiv. Das Semicarbazon (3) zeigt eine

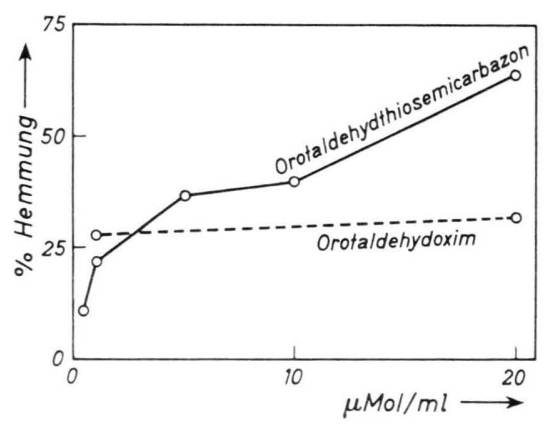

Abb. 1. Hememnde Wirkung des Orotaldehydoxims und des Orotaldehydthiosemicarbazons auf Neurosporat crassa. Die Hemung ist nach 100 -stdg. Inkubation bei $25^{\circ}$ gemessen.

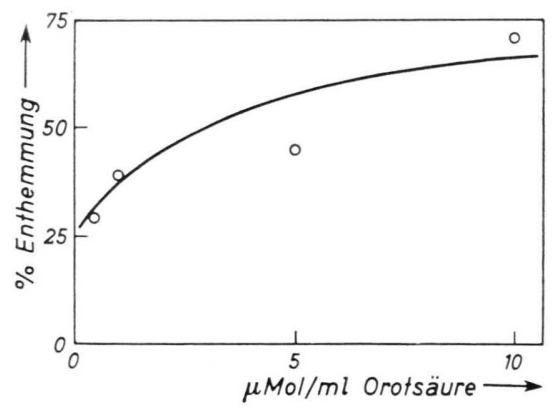

Abb. 2. Enthemmende Wirkung der Orotsäure auf den wuchshemmenden Effekt des Orotaldehydoxims. Die Enthemmung ist nach 100-stdg. Inkubation bei $25^{\circ}$ gemessen.

${ }^{4}$ H. M. Rauen, G. Hess u. J. Merchery, Hoppe-Seyler's Z. physiol. Chem. 315, 60 [1959]; H. M. Rauen u. G. Hess, 317,10 [1959] ; H. M. Rauen u. R. Nonhof, Arzneimittelforsch. 13, 558 [1963].

* Diesen Stamm verdanken wir der Freundlichkeit von Herrn Prof. Dr. H. M. Rauen (Physiologisch-Chemisches Institut der Universität Münster in Westfalen). 
10-proz. $(20 \mu \mathrm{Mol} / \mathrm{ml})$, das Phenylhydrazon (4) eine 15-proz. ( $20 \mu \mathrm{Mol} / \mathrm{ml})$ Hemmung an.

Auf der Abb. 2 ist die enthemmende Wirkung der Orotsäure auf das Orotaldehydoxim zu sehen. Die Wirkung des Thiosemicarbazons (2) wird bis zu $80 \%$ von der Orotsäure in einer Konzentration von $10 \mu \mathrm{Mol} / \mathrm{ml}$ enthemmt.

Aus diesen Ergebnissen folgt, daß die untersuchten Orotaldehyd-Derivate als Orotsäureantagonisten bei Neurospora wirken. In dieser Hinsicht stellen diese Verbindungen eine neue Gruppe von Orotsäure-Antimetaboliten dar.

Weitere Untersuchungen über die biologische, insbesonders die cytostatische Wirkung und den biochemischen Wirkungsmechanismus der OrotaldehydDerivate sind im Gange.

\title{
Vermehrte RNS-Synthese in Makrophagen während der Phagocytose antigenen Materials
}

\begin{abstract}
Increased RNA Synthesis in Macrophages during Phagocytosis of Antigenic Materials
KLAUS-UlRich HaRTMANN

Max-Planck-Institut für Virusforschung, Abteilung für physikalische Biologie, Tübingen

(Z. Naturforschg. 24 b, 1317-1323 [1969]; eingegangen am 2. April 1969)

Macrophages have been cultured as uniform cell populations. These cells incorporate uridine but not thymidine into their nucleic acids during the culture period. Sheep erythrocytes in the presence of macrophage cytophilic antibodies were agglutinated to rosettes and phagocytized. Cytophilic antibodies did not have to be species specific; phagocytosis was not complement-dependant. During phagocytosis, incorporation of $\mathrm{H}^{3}$-uridine into the RNA was increased. Sucrose gradient analysis showed that the increase in uridine incorporation was mainly due to increased m-RNA synthesis.
\end{abstract}

Makrophagen sind Zellen, welche in höheren Tieren allgegenwärtig sind, als Monocyten im Blut, als Histiocyten im Gewebe, als Kupffer sche Sternzellen in der Leber usw. Diese Zellen sind in der Lage, antigenes Material zu erkennen und zu phagocytieren ${ }^{1}$. Eine Reihe von Experimenten ${ }^{2-7}$ wurde interpretiert, daß die Makrophagen eine wichtige Rolle, eine essentielle Rolle, bei der Erkennung und Verarbeitung des immunogenen Materials und bei de Auslösung der Antikörpersynthese ausführen; die Erklärung dieser Befunde ist nicht allgemein anerkannt.

In der vorliegenden Arbeit wird die Aufnahme von Antigen (Erythrocyten) und die gleichzeitige Synthese von RNS untersucht. Die Makrophagen waren als eine sehr einheitliche Zellsuspension aus

1 E. B. H. Mudd and S. Mudd, J. gen. Physiol. 16, 625 [1933].

2 M. Fishman, J. exp. Medicine 114, 837 [1961].

3 F. L. Adler, M. Fishman, and S. Dray, J. Immunology 97, 554 [1966].

4 D. C. Bishop, A. V. Pisciotta, and P. Abramoff, J. Immunology 99, 751 [1967].

5 D. Jacherts and H. Noltenius, Z. med. Mikrobiol. Immunology 152, 112 [1966].
Peritonealhöhlen nicht-vorbehandelter Mäuse entnommen ${ }^{8}$ und in Leigh to n - Röhrchen kultiviert worden. Schaferythrocyten (SRBK) als Antigen wurden zugesetzt; um die Anheftung des Antigens und optimale Phagocytose zu fördern, war in vielen Experimenten Antiserum gegen SRBK, welches makrophagen-cytophile Antikörper enthielt ${ }^{9}$, zugesetzt worden. Dabei spielten die Überlegungen von JERNE ${ }^{10}$ eine Rolle, daß zirkulierende Antikörper bereits zur ersten Erkennung des Antigens anwesend sein sollten.

Bereits kurze Zeit nach Zugabe von Antigen und cytophilem Antikörper bilden sich um die Makrophagen Rosetten von Erythrocyten; binnen weniger Stdn. werden diese phagocytiert. In dieser Zeit wird vermehrt $\mathrm{H}^{3}$-Uridin in die RNS der Makrophagen

\footnotetext{
6 B. A. Askonas and J. M. Rhodes, Nature [London] 205, 470 [1965].

7 A. A. Gottlieb, V. R. Glišin, and P. Doty, Proc. nat. Acad. Sci. USA 57, 1849 [1967].

8 J. L. Whitby and D. Rowley, Brit. J. exp. Pathol. 40, 358 [1959].

9 S. V. Boyden, Immunology 8, 474 [1964].

10 N. K. JERNE, J. Immunology 76, 209 [1956].
} 